\title{
Concurrent Voltage Control and Dispatch of Active Distribution Networks by means of Smart Transformer and Storage
}

\author{
Xiang Gao, Student Member, IEEE, Fabrizio Sossan, Member, IEEE, Kostantina Christakou, Member, IEEE, \\ Mario Paolone, Senior Member, IEEE, Marco Liserre, Fellow, IEEE.
}

\begin{abstract}
Dispatching active distribution networks is expected to play an important role in the smart grid technologies. Voltage control is also starting to be widely proposed to avoid voltage violations in the electrical grid. The integration of the storage in the so called smart transformer (ST), which is a solid state transformer with control and communication functionalities, can help combine both the services. The added value of such a configuration is that it allows the full decoupling of the reactive power flows between the medium voltage (MV) and low voltage (LV) networks. We show the augmented flexibility of such a configuration by proposing a control strategy where, $i$ ), the storage system is used to achieve dispatched-by-design operation of the LV network active power flow, and, ii), the two ST power converters to control the voltage in both the MV and LV grid on a best effort basis. The control strategy is validated by simulations using the IEEE 34 nodes MV test feeder and the Cigre LV reference network. Moreover, control performance is benchmarked against a conventional network setup, where the BESS is connected to the LV network through a DC/AC power converter and the ST transformer is replaced by a conventional transformer.
\end{abstract}

Index Terms-BESS, renewable energy, smart transformer, voltage regulation, dispatch-by-design

\section{INTRODUCTION}

$\mathbf{T}$ HE concept of Active Distribution Networks (ADNs) refers to monitored electrical grids where distributed energy resources, like Distributed Generation (DG), storage, and flexible loads, are controlled by a suitable Energy Management System (EMS) to achieve specific grid objectives, see e.g., [1], [2]. Control objectives usually refer to optimal voltage control, management of line congestions, local load balance, losses minimization and self consumption [3]-[5].

In this respect, the use of battery energy storage systems (BESSs), traditionally considered for microgrid applications to store excess renewable generation, see e.g. [6], [7], is gaining

Xiang Gao and Marco Liserre are with the Chair of Power Electronics, Christian-Albrechts-Universität zu Kiel, Germany, e-mail: $\{x g$ ml\}@tf.uni-kiel.de. Fabrizio Sossan, Kostantina Christakou, and Mario Paolone are with the Distributed Electrical Systems Laboratory, École Polytechnique Fédérale de Lausanne, Switzerland (EPFL), e-mail: \{fabrizio.sossan, mario.paolone\}@epfl.ch.

This work was supported by the Vaud Canton of Switzerland within the project "500 kWh Pilot Energy Storage System for the Control of Distribution Systems with Dispersed Generation", the European Research Council under the European Union's Seventh Framework Program (FP/2007-2013) / ERC Grant Agreement n. [616344] - HEART, and European Union/Interreg V-A—Germany-Denmark, under PE:Region Project. increased focus even in the context of large interconnected power systems thanks to their decreasing cost, longer life span and technical progression of associated technologies, see e.g [8], [9]. In the existing technical literature, BESSs have been proposed in applications to provide ancillary services to the grids, like voltage support and primary frequency regulation [10]-[13], improvement of self consumption [14], load peak shaving [15], and dispatch of aggregated stochastic renewable generation and prosumption [16]-[22].

Another key technology which is expected to enable the integration of increased amounts of renewable generation in distribution systems is the smart transformer (ST). An ST is a three-stage power electronics transformer that interfaces the voltage levels of MV and LV distribution systems [23], [24] and makes available the DC grid connection [25]. On top of adapting the voltage levels, the ST decouples the reactive power flows of the two electric networks [26], [27], therefore adding an additional degree of freedom which can be exploited in ADNs control strategies.

Whereas the conventional configuration of grid-connected BESS consists in connecting the battery DC side to the $\mathrm{AC}$ side through a DC/AC power converter, we consider in this paper the case where the battery is connected to the ST DC bus. We call this configuration ST with integrated storage. With respect to a configuration with a conventional transformer (CT) and grid-connected BESS, the considered configuration has the advantage of fully decoupling the flow of the MV and LV networks in terms of both active and reactive power.

This paper proposes a control strategy for a ST with integrated storage which has the following concurrent objectives: i) dispatching the active power flow of the underneath distribution system (composed of stochastic prosumption) according to a profile established the day before the operation, and, ii) providing voltage control on a best effort basis to both the LV network and the upper-stream MV distribution network. As detailed later, the first control objective is achieved by exploiting the design proposed in [18]. It consists in determining, the day before operation, a dispatch plan at 5-minute resolution by using prosumption forecasts and accounting for the information on the residual BESS charge. The dispatch plan is then tracked in real-time by controlling the BESS power injection. The latter control objective is achieved by controlling the reactive power of the MV converter, for the case of the MV grid, and, for the LV grid, using the LV converter to set a proper voltage level at the root of the 
feeder such that voltage deviations from the reference value are minimized along the feeder. The resolution of both control actions is 10 seconds. Voltage control exploits the knowledge of the grid state (voltage magnitude and power injections), which can be provided by remote terminal units, phasor measurement units (PMUs) or smart meters coupled with state estimation processes, see e.g. [28].

The performance of the proposed control strategy is verified by simulations by considering the IEEE 34 nodes test feeder, where one of the MV nodes is replaced by the radial LV CIGRE reference network. The considered configuration (ST with integrated storage) is compared against a conventional configuration, where the two same networks are interfaced by a CT and the LV network is equipped with a grid-connected BESS installed at the grid connection point.

This paper is organized as follows: Section II and Section III describe the two case studies considered in this paper, introduces the notation and describes the formulation of the control problems; Section IV and Section V describe the simulation setup and shows the simulation results. Finally, Section VI summarizes the main contributions of this work and states the conclusions.

\section{NETWORK TOPOLOGY AND NOTATION}

\section{A. Description of the considered network topologies}

A MV/LV distribution system with the following two configurations is considered:

- Case 0 (Fig. 1): The interface between the MV and LV level is a conventional transformer. The storage capacity is installed at the LV level at the root of feeder and gridconnected with a DC/AC power converter, controllable with active and reactive power set-points. The MV and LV systems are decoupled in terms of active power within the limit imposed by the storage energy/power capacities. This case represents the typical approach for interfacing BESS in distribution networks and is used as a benchmark case against which we compare the performance of Case 1. The control objectives are to provide dispatchedby-design operation of the active power flow and voltage regulation on a best effort basis to the MV and LV level.

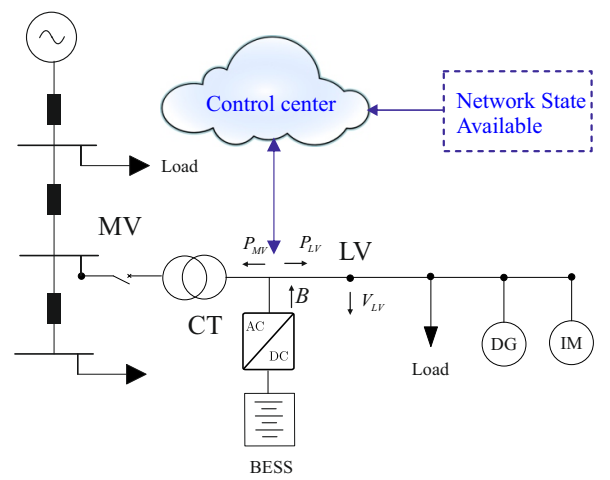

Fig. 1. Case 0. From the left to the right: a generic MV network, a conventional transformer (CT) and a generic LV network.

- Case 1 (Fig. 2): The interface between MV and LV is a ST and the storage capacity is connected to the
ST DC bus. With respect to Case 0, this configuration requires two power converters, which are to be sized for the rated power of the LV network. Similarly to Case 0, the control objectives are dispatched-by-design operation of the active power flow and voltage regulation of the LV and MV network but in a separate fashion. Voltage regulation is expected to be more effective in this case because the power flows between the two networks are completely decoupled.

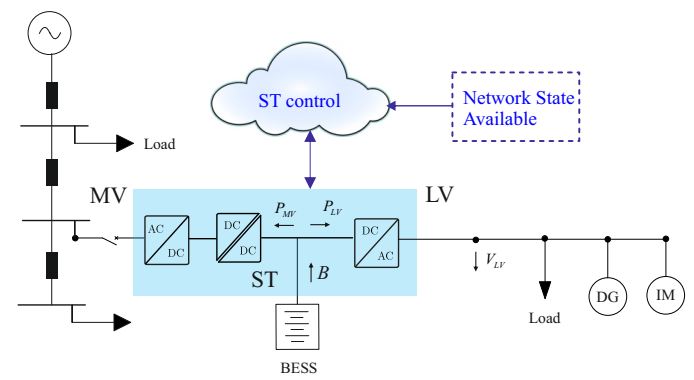

Fig. 2. Case 1. From the left to the right: a generic MV network, a smart transformer (ST) with integrated storage and a generic LV network.

Voltage control in both Case 0 and Case 1 requires the knowledge of the voltage levels of the MV and LV buses, which are assumed known from, for example, PMU-based and smart meters-based monitoring infrastructures and integrated with state estimation algorithms in case of non complete observability, see e.g., [28], [29].

\section{B. Notation and working hypotheses}

We consider a MV and LV network with $M$ and $L$ buses respectively. We assume that both networks are balanced. Therefore the notation for voltages, currents and power flows refers to the direct sequence (a way to extend the proposed method to unbalanced grids is discussed in the following Appendix A). The voltage magnitude at a given bus $m=$ $1, \ldots, M$ of the MV network is denoted by $V_{\mathrm{MV}, m}$, whereas it is $V_{\mathrm{LV}, l}, l=1, \ldots, L$ for the $\mathrm{LV}$ buses $l=1, \ldots, L$. The complex power injections are $P_{\mathrm{MV}, m}+j Q_{\mathrm{MV}, m}$ and $P_{\mathrm{LV}, l}+j Q_{\mathrm{LV}, l}$ for $\mathrm{MV}$ and $\mathrm{LV}$ network, respectively. The quantity $V^{*}$ denotes the direct sequence voltage of the slack bus for both grids.

\section{Problem Formulation}

In this section, we first describe the formulation of the control methods for Case 1, which is the major contribution of the paper. Based on this, we therefore derive the formulation for Case 0.

\section{A. Case 1}

The problem we tackle is determining the real-time active and reactive power set-points of the MV converter and voltage set-points of the LV converter such that:

1) the active power flow on the MV side tracks the socalled dispatch plan, i.e., a sequence of average power 
consumption profile at 5-minute resolution established the day before operation [18];

2) the reactive power injection of the MV converter is controlled to provide voltage regulation support to the MV network;

3) the voltage reference of the LV converter is controlled to provide voltage regulation to the LV network.

1) Control Problem of the $M V$ power converter: It consists in a hierarchical control strategy where the active and reactive power injections are determined sequentially and coupled by the apparent power constraint of the converter, as described in the following.

a) Active power set-point: First, the active power injection $P_{\mathrm{MV}}$ ST at the ST bus is controlled to dispatch the operation of the underneath distribution system according to the control process described in [18]. In other words, it is required that the active power flow at the ST bus follows a sequence of average power consumption values at 5-minute resolution, called dispatch plan and denoted by the sequence $\widehat{P}_{1}, \ldots, \widehat{P}_{288}$, established the day before the operation. Although not being a contribution of this paper, the main features and operating principle of this control design are summarized in the following for the sake of clarity and to enable results reproducibility. Nevertheless, for a thoughtful tractation, the reader is referred to [18]. The dispatch plan (which in this work is assumed given) consists in the sum of two terms: the expected value of the demand and distributed local generation (simply referred to as prosumption) and the offset profile, which has the objective of incorporating into the dispatch plan an additional power flow (positive or negative) necessary to restore a suitable level of flexibility for the incoming day of operation. The former component is generated by applying a prediction tool, while the latter by a robust or scenariobased optimization problem which enforces battery energy constraints by leveraging the knowledge of the prediction uncertainties.

For the considered case in Fig. 2, the injection $P_{\mathrm{MV}, \mathrm{ST}, k}$ at the ST bus at the discretized time interval $k$ is the algebraic sum between the active power realization $P_{\mathrm{LV}, \mathrm{ST}, k}$ of the underneath distribution system and the storage charge/discharge value $B_{k}^{o: 1}$

$$
P_{\mathrm{MV}, \mathrm{ST}, k}=P_{\mathrm{LV}, \mathrm{ST}, k}+B_{k}^{o} \text {. }
$$

The latter component $B_{k}^{o}$ is the controlled quantity. It is computed with a model predictive control algorithm, proposed and experimentally validated in [18] also, which has the objective of minimizing the mismatch between the 5-minute average active power flow realization and the dispatch plan value for the current 5-minute interval, while obeying to battery constraints. The average power flow at the ST bus, denoted $\bar{P}_{\mathrm{MV}, \mathrm{ST}, k}$, is calculated over a fixed 5-minute horizon (from $\underline{k}$ to $\bar{k}$, which respectively denote the first and last $k$-index of the current 5-minute period) by using the progressively available measurements from the sensing device at the grid connection point. The current 5-minute set-point from the

\footnotetext{
${ }^{1}$ At this stage, we assume that losses in the power conversion stages are negligible [30].
}

dispatch plan is denoted by $\widehat{P}_{k}$. The model predictive control (MPC) action is recomputed at each 10 seconds time interval: at each iteration, the MPC determines the control trajectory for the remaining 5-minute horizon from, $k$ to $\bar{k}$, and only the first set-point $B_{k}^{o}$ is actuated and other discarded. The reason in favour of MPC rather than a conventional feedback control loop is that, since the control objective is accomplishing a certain energy value in an assigned time frame (i.e. the battery energy throughput compensates for the 5-minute average error between the realization and dispatch plan), MPC allows to choose the power trajectory which is respectful of the battery operational constraints. The average battery power injection in the current fixed 5-minute interval from $\underline{k}$ to $\bar{k}$ is

$$
\bar{B}\left(B_{\underline{k}}^{o}, \ldots, B_{\bar{k}}^{o}\right)=\frac{1}{30} \sum_{j=\underline{k}}^{\bar{k}} B_{j}^{o},
$$

where 30 is the number of 10 seconds intervals in the 5-minute period. The MPC problem consists in finding the BESS power sequence $\boldsymbol{B}_{k}^{o}=\left[B_{k}^{o}, \ldots, B_{\bar{k}}^{o}\right]$ that minimizes the squared mismatch between the average power flow at the ST bus $\bar{P}_{\mathrm{MV}, \mathrm{ST}, k}+\bar{B}(\cdot)$ and the dispatch set-point $\widehat{P}_{k}$, while being subject to BESS DC voltage, DC current, and SOC constraints. Formally the problem is:

$$
\boldsymbol{B}_{k}^{o}=\underset{\boldsymbol{B}_{k}=\left[B_{k}, \ldots, B_{\bar{k}}\right]}{\arg \min }\left\{\left(\bar{P}_{\mathrm{MV}, \mathrm{ST}, k}+\bar{B}\left(\boldsymbol{B}_{k}\right)-\widehat{P}_{k}\right)^{2}\right\}
$$

subject to

$$
\begin{aligned}
& v_{\min } \preccurlyeq V\left(\boldsymbol{B}_{k}, v_{k}\right) \preccurlyeq v_{\max } \\
& i_{\min } \preccurlyeq I\left(\boldsymbol{B}_{k}, v_{k}\right) \preccurlyeq i_{\max } \\
& i_{\min } \preccurlyeq \operatorname{SOC}\left(\boldsymbol{B}_{k}, v_{k}\right) \preccurlyeq i_{\max },
\end{aligned}
$$

where $V(\cdot), I(\cdot), \mathrm{SOC}(\cdot)$ are prediction models of the battery voltage on the DC bus, DC current and state of charge, respectively, and $\left(v_{\min }, v_{\max }\right),\left(i_{\min }, i_{\max }\right)$ and $\left(\mathrm{SOC}_{\min }, \mathrm{SOC}_{\max }\right)$ are the respective allowed bounds ( $\preccurlyeq$ denotes the componentwise inequality). The optimization problem in (3)-(6) is non convex due to the presence of battery DC voltage and current constraints, which are non convex in the decision variable $\boldsymbol{B}$ even for battery models composed by the well known two time constants circuit model. For the simulation results proposed in this paper, we adopt the convex equivalent formulation of (3)-(6) proposed in [18] (convenient because it is more tractable and admits a global optimum, if it exists), which drops the decision variable $\boldsymbol{B}$ in favour of the battery DC current. In this new framework, DC current, voltage and SOC constraints can be expressed as a linear combination of the decision variables, which, coupled to a linear cost function, lead to a convex formulation of the problem. As detailed in [18], voltage constraints are enforced by using a two time constant model, whereas the SOC is an integral model of the current. The models, both structure and parameters, used for the simulations of this paper are the same as in [18] and were identified from experimental data.

It is worth noting that the capability of compensating for the mismatch between realization and dispatch plan depends on the battery power rating and energy capacity, which is assumed 
of the proper size to absorb the typical dispatch forecasting errors for the portion of the network under consideration. ${ }^{2}$

b) Reactive power set-point: Once the active power injection $P_{\mathrm{MV}, \mathrm{ST}}$ has been determined, the reactive power injection $Q_{\mathrm{MV}, \mathrm{ST}}$ of the power converter is controlled in order to provide primary voltage control to the MV grid. It is computed by solving a constrained optimization problem which minimizes the voltage deviations of the network buses from the network rated value (1 p.u.). At each time-step the state of the network, i.e., the phase-to-ground voltage phasors is known thanks to measurements or state estimation processes. Using this information, we can compute the voltage sensitivity coefficients of all the network nodes with respect to absorbed/injected reactive power of the BESS:

$$
K_{Q, m}:=\frac{\partial V_{\mathrm{MV}, m}}{\partial Q_{\mathrm{MV}, \mathrm{ST}}}, \quad m=1, \ldots, M .
$$

e.g., by solving the linear system of equations presented in [31]. At this stage, thanks to using the sensitivity coefficients, we compute the optimal required reactive power adjustments $\left\{\Delta\left(Q_{\mathrm{MV}, \mathrm{ST}}\right)^{*}\right\}$ which lead to the desired operational set-point for voltage control via the following constrained optimization problem:

$$
\min _{\Delta Q_{\mathrm{MV}, \mathrm{ST}}}\left\{\sum_{m=1}^{M}\left(V_{M V, m}+K_{Q, m} \Delta Q_{\mathrm{MV}, \mathrm{ST}}-V^{*}\right)^{2}\right\}
$$

subject to

$$
P_{\mathrm{MV}, S T}^{2}+Q_{\mathrm{MV}, \mathrm{ST}}^{2} \leq S_{\mathrm{nom}}^{2}
$$

where $S_{\text {nom }}$ is the nominal apparent power of the MV converter. Its value is normally a dynamic function of the converter operating conditions, like the terminal voltage on the AC side. At this stage, we consider it constant, and the inclusion of more representative models will be considered in future work.

2) Control Problem on the LV side : The ST allows to provide primary voltage control to the LV grid by setting the voltage reference at the root of the feeder to assure suitable voltage levels along the network buses. In order to do so, we compute the direct sequence voltage sensitivity coefficients of all the network nodes with respect to the reference voltage of the LV grid:

$$
K_{V^{*}, m}:=\frac{\partial V_{L V, m}}{\partial V^{*}}, \quad m=1, \ldots, L .
$$

Sensitivity coefficients in (10) can be obtained by solving a linear system of equations similar to the case of voltage sensitivities with respect to transformer's tap changers positions (e.g., section II.C in [31], or [32] for another example). Once the coefficients are known, the suitable voltage level of the slack bus is determined by the following optimization problem:

$$
\min _{\Delta V^{*}}\left\{\sum_{m=1}^{M}\left(V_{L V, m}+K_{V^{*}, m} \Delta V^{*}-V^{*}\right)^{2}\right\} .
$$

The computation of the sensitivity coefficients in (10) requires the knowledge of the voltages, which are assumed known from, e.g., smart meters readings or a state estimation process.

\footnotetext{
${ }^{2}$ The sizing aspect is beyond the scope of this paper.
}

\section{B. Case 0}

The control variables are the active $P_{\mathrm{BESS}}$ and reactive power $Q_{\mathrm{BESS}}$ injection of the BESS converter. The former is controlled such that the average aggregated power flow $\bar{P}_{\mathrm{CT}, \mathrm{MV}}$ at the CT bus bar follows the dispatch plan. The control problem is equivalent to what discussed for (3)-(5), unless for an additional constraint to enforce the BESS active power flow within the limits of the BESS DC/AC converter.

Once $P_{\mathrm{BESS}}$ is known, the reactive power injection $Q_{\mathrm{BESS}}$ is determined as deviation $\Delta Q_{\mathrm{BESS}}$ from the current operating point in order to provide voltage regulation to the buses of both MV and LV networks. The control problem is as:

$$
\begin{aligned}
\min _{\Delta Q_{\mathrm{BESS}}} & \left\{\sum_{m=1}^{M}\left(V_{M V, m}+K_{Q, m} \Delta Q_{\mathrm{BESS}}-V^{*}\right)^{2}+\right. \\
& \left.+\sum_{l=1}^{L}\left(V_{L V, l}+K_{Q, l} \Delta Q_{\mathrm{BESS}}-V^{*}\right)^{2}\right\}
\end{aligned}
$$

subject to

$$
P_{\mathrm{BESS}}^{2}+Q_{\mathrm{BESS}}^{2} \leq S_{\mathrm{nom}}^{2},
$$

where $K_{Q, m}$ and $K_{Q, l}$ are the sensitivity coefficients of the voltage levels of the MV and LV buses, respectively, with respect to the BESS reactive power injection. It is noteworthy that in the formulation above we do not enforce BESS energy constraints because the control only involves reactive power and the converter can operate as a static VAR compensator. As mentioned earlier, in Case 0 , the networks are not decoupled in terms of reactive power flow, in other words the optimization problem (12)-(13) aims at finding the best compromise in terms of voltage levels for both the LV and MV networks. Indeed, we expect this regulation to be less effective than Case 1.

\section{Simulations METHODS}

\section{A. Simulation Setup}

The simulation setup consists in the IEEE 34 nodes MV reference network [33]. All the nodes are simulated in terms of aggregated active/reactive power injections, unless for one node which is simulated into details by using the CIGRE LV benchmark grid [34]. Such a grid is interfaced with the MV level through a CT or ST to represent Case 0 and Case 1, respectively, as described in II. The location of the node with the detailed model of the LV part is chosen as the one with the largest reactive power sensitivity coefficient, such that its support to the MV network voltage regulation through the ST or BESS converter is the most effective. The relevant inputs for the networks simulations are:

- For the MV grid: reference profiles are according to experimental measurements from a test network of compatible size, except for the $\mathrm{MV} / \mathrm{LV}$ interface, where it is as the dispatch plan. All loads are unbalanced.

- For the LV grid: the active power flow injections at the buses are obtained starting from the time varying profile proposed by CIGRE network specifications scaled down according to the nominal power of each node. Reactive 
flows are calculated assuming a constant power factor (0.9). The peak value of the load in whole LV grid is $150 \mathrm{kVA}$. The dispatch plan at the MV/LV interface (which is normally generated by integrating forecast of the prosumption, as explained in the foregoing) is given by the sum of the single injections at the buses plus a random component to simulate forecast uncertainties. Loads are assumed balanced.

The behavior of the ST with integrated storage is modeled in terms of the power flow injections to the MV and LV networks. The power converter on the MV side is controlled by enforcing active/reactive power references (determined as discussed in III-A1), while the LV power converter is controlled to keep a certain voltage level, which is given as described in III-A2. The control logic of power converters is not simulated. At this stage, the efficiency of the power conversion is assumed unitary. ${ }^{3}$ Both MV and LV networks are simulated via power flows analysis. The BESS energy capacity is assumed given and is $500 \mathrm{kWh}$. For Case 0, the BESS converter nominal power is $720 \mathrm{kVA}$, whereas, for Case 1 , the nominal power of $\mathrm{MV}$ and LV ST converters is $720 \mathrm{kVA}$.

\section{B. Demand and Scenarios}

Two scenarios of the aggregated power flow profile at the $\mathrm{MV} / \mathrm{LV}$ interfaced are considered. Both scenarios originate from experimental measurements of the electrical flow of a group of buildings equipped with rooftop PV installations with comparable power rating as the considered LV benchmark network. Scenarios, are visible in Fig. 3, consist in two daily profiles, and correspond to a weekday and to a weekend day. In the latter, the decrease of the demand due to PV generation is well visible in the central part of the day.

\section{Performance Metrics}

To measure the performance of the dispatch-by-design strategy, we evaluate the accumulated absolute value of the deviation between the active power flow realization $P(t)$ and dispatch plan $\widehat{P}(t)$ and time $t$ :

$$
m_{0}=\sum_{t=0}^{T}|\widehat{P}(t)-P(t)|
$$

which is an indicator of the amount of imbalances generated by this portion of the network. Voltage control performance are measured by comparing the mean $\bar{m}_{1}$, and maximum $m_{1}^{\uparrow}$ of the voltage deviations of the buses with respect to the reference value 1 p.u. With reference to the MV network, we denote with $\mathcal{D}$ the set of voltage deviations for all the buses $l=1, \ldots, M$ and time intervals $t=1, \ldots, T$ :

$$
\mathcal{D}=\left\{\left(V_{\mathrm{MV}, l}(t)-1\right), t=1, \ldots, T, l=1, \ldots, M\right\} .
$$

The formal definitions of the two metrics for the voltage (previously defined in a verbose manner) are:

$$
\begin{aligned}
& \bar{m}_{1}=\frac{1}{T} \frac{1}{L} \sum_{d \in \mathcal{D}}|d| \\
& m_{1}^{\uparrow}=\max \quad\{|d|, \forall d \in \mathcal{D}\} .
\end{aligned}
$$

${ }^{3}$ This is reasonable approximiation since from experimental measure
For the LV network, the computation is analogue and obtained by considering in the set $\mathcal{D}$ the voltage levels of the LV buses.

\section{RESULTS AND DISCUSSION}

Two mainstream analysis, A and B, are carried out: (A) an assessment and comparison of the control performance of Case 1 and Case 0 ; (B) the components of Case 0 are sized in order that the voltage control performance in Case 0 matches Case 1. In other words, the proper size of a CT and BESS converter, which can achieve the same performance as the ST, is evaluated: the objective here is establishing which among the two setups achieves smaller components sizes. The latter analysis does not include evaluating the dispatch plan tracking performance because this depends uniquely on the battery size, which is the same in the two cases. The time resolution for the first set of simulations is 10 seconds. For the second set, the suitable sizes of CT and converter are inferred by using simulations at 300 seconds resolution while trying different combinations of size. Once the suitable sizes are determined, simulations at 10 seconds resolution are performed and benchmarked against the other cases.

\section{A. Control performance comparison: Case 1 vs Case 0}

The results of the active power control in Case 1 for Scenario 1 and 2 are shown in Fig. 3, which features the dispatch plan, the active power flow realization at ST MV/LV interface (therefore including the storage action) and at the ST LV side. As visible, the realization at the MV/LV interface tracks the dispatch plan, thus achieving dispatched-by-design operation of the active power flow, while the realization at the LV side (thus without battery injection) does not always match the dispatch plan due to forecasting errors.
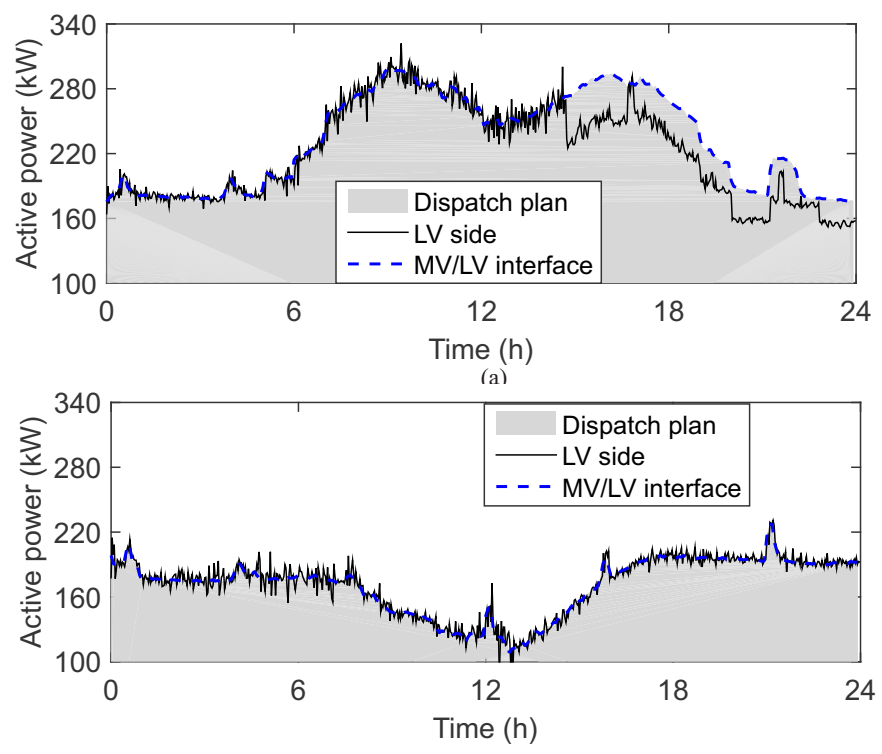

(b) 2

Tracking performance is summarized in Table I, which shows equivalent performance between Case 0 and Case 1 
(indeed, they both achieve dispatched-by-design operation) and substantially better performance of when no control action is performed.

TABLE I

Dispatch PLAN TRACKING PERFORMANCE

\begin{tabular}{|l|l|c|c|c|}
\hline & Metric & Case 0 & Case 1 & No Control \\
\hline S1 & $m_{0}(\mathrm{~kW})$ & 0.16 & 0.16 & 11.07 \\
S2 & $m_{0}(\mathrm{~kW})$ & 0.23 & 0.23 & 0.27 \\
\hline
\end{tabular}

As far as the voltage control is concerned, the metrics of voltage variations for the first and second scenarios are summarized in Table II with the respect to 1 p.u. of the voltage. To compare the improvement of both cases, for Scenario 1, the mean and maximum voltage variations in Case 1 are reduced by $14.1 \%$ and $9.3 \%$, respectively, with respect to Case 0 in the MV grid, and by $69.2 \%$ and $60.5 \%$ in the LV. It is worth noting that in Case 0, the voltage variations in LV grid can be higher than $10 \%$ of the nominal value, thus exceeding the operational limits of voltage regulation for LV systems. For Scenario 2, the mean and maximum voltage variations in Case 1 are reduced by $11.4 \%$ and $8.0 \%$ thanks to using the ST. In LV grid, the ST achieves the same improvement as for Scenario 1, and it can be seen that without ST, it is not possible to regulate voltage variations in LV grid within the prescribed $\pm 10 \%$ interval.

TABLE II

Voltage Control Performance

\begin{tabular}{|l|c|c|c|c|}
\hline Metric & Case 0 MV & Case 1 MV & Case 0 LV & Case 1 LV \\
\hline \multicolumn{5}{|c|}{ Scenario 1 } \\
\hline $\bar{m}_{1}$ & $0.78 \%$ & $0.67 \%$ & $2.76 \%$ & $0.85 \%$ \\
$m_{1}^{\uparrow}$ & $3.35 \%$ & $3.04 \%$ & $11.48 \%$ & $4.53 \%$ \\
\hline \multicolumn{5}{|c|}{ Scenario 2 } \\
\hline $\bar{m}_{1}$ & $0.79 \%$ & $0.7 \%$ & $2.76 \%$ & $0.85 \%$ \\
$m_{1}^{\uparrow}$ & $3.38 \%$ & $3.11 \%$ & $11.47 \%$ & $4.53 \%$ \\
\hline
\end{tabular}

The distribution of the phase-to-ground voltages along the MV nodes at a specific time instant (when the maximal voltage variation in MV grid in Case 0 occurs) is is shown in Fig. 4 for both Case 0 and Case 1, and Scenario 1 and Scenario 2. Results shows that for both scenarios, the ST controlled with the proposed control strategy allows achieving voltage levels closer to the unit value with respect to the case with the CT.

The evolution in time of the voltage at the MV/LV interface is shown in Fig. 5 for both Scenario 1 and 2. It can be seen that the voltage level in Case 1 is substantially improved with respect to Case 0.

Fig. 6 shows the reactive power injection of the ST MV power converters and BESS power converter in both scenarios. It can be seen that with the same size, the ST is able to inject more reactive power thanks to the decoupled architecture.

The voltage variations along the $\mathrm{LV}$ grid buses for Case 0 and Case 1 are shown in Fig. 7 by means of boxplots, featuring the mean, standard deviation and outliers. For Case 1, the voltage values for Scenario 1 and 2 are equivalent, therefore they are shown only once in Fig. 7(c). This denotes that the ST is able to keep the same performance, regardless of

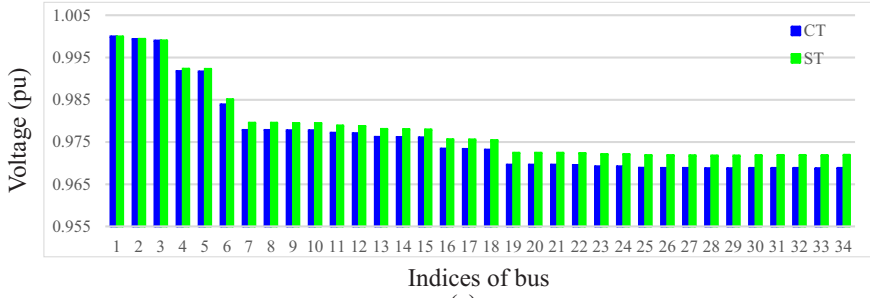

(a)

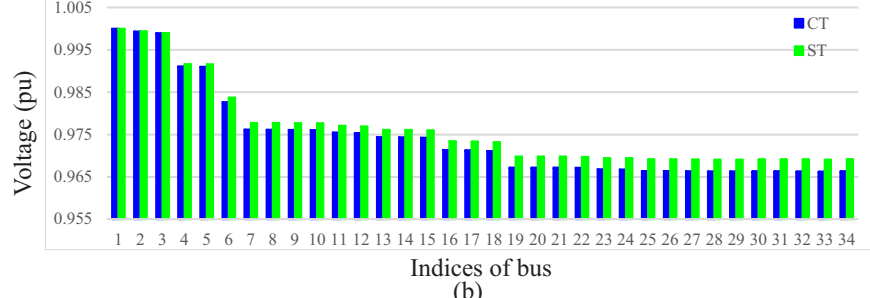

(b)

Fig. 4. MV grid voltage profile (Phase C) (a). Scenario 1, (b). Scenario 2

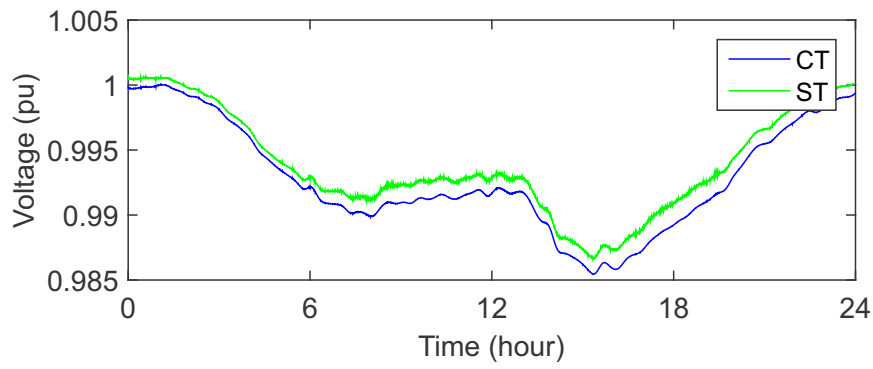

(a)

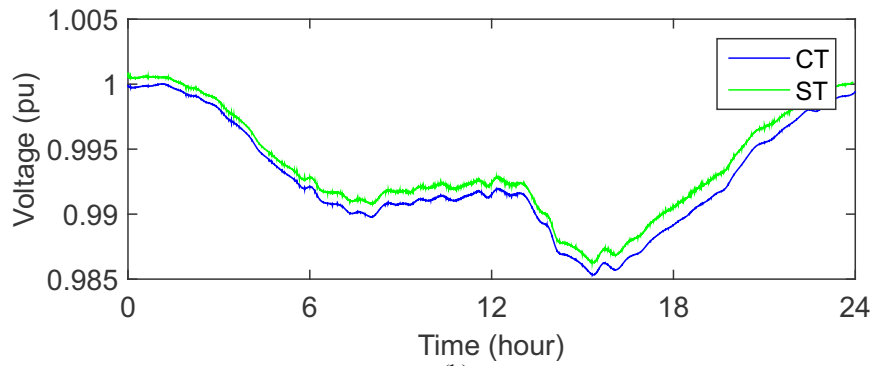

(b)

Fig. 5. Daily voltage profile of Bus 16 (Phase B) in MV grid (a). Scenario 1, (b). Scenario 2

the scenario. From Fig. 7, it is visible that the ST improves the voltage performance in the LV grid in both scenarios. In particular, it increases the voltage level at the MV/LV interface (bus 1) such that the total variation from the reference value along the feeder is reduced.

\section{$B$. Sizing of the systems to match performance}

The objective of this analysis is sizing the component of Case 0 (CT and BESS DC/AC power converter) to match the control performance of Case 1. Therefore, it allows to establish which size of the components for the conventional configuration case achieves the same results of the ST case. The results are presented in Fig. 8 for the MV and Fig. 9 for the LV grid, where the horizontal plane shows the performance of the ST and each bar the one for a specific combination of 


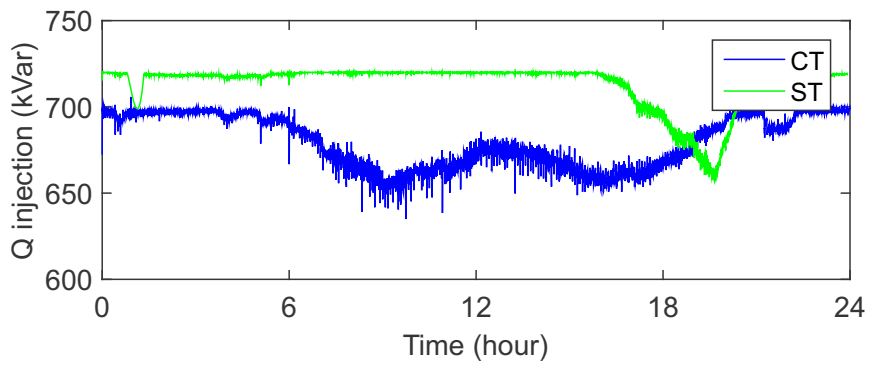

(a)

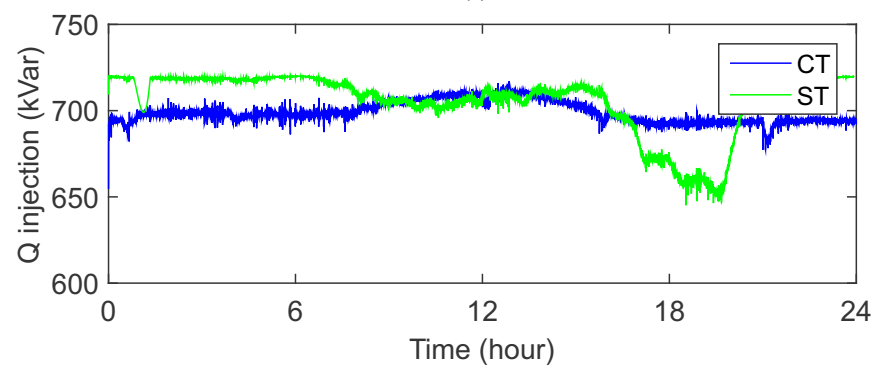

(b)

Fig. 6. Reactive power injection vs. time (a). Scenario 1, (b). Scenario 2

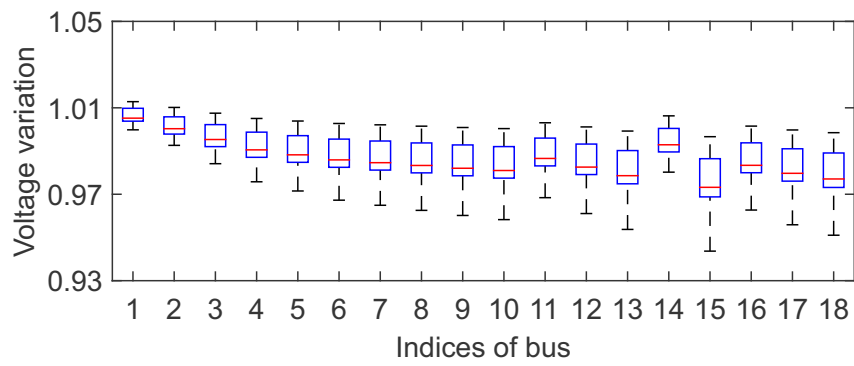

(a)

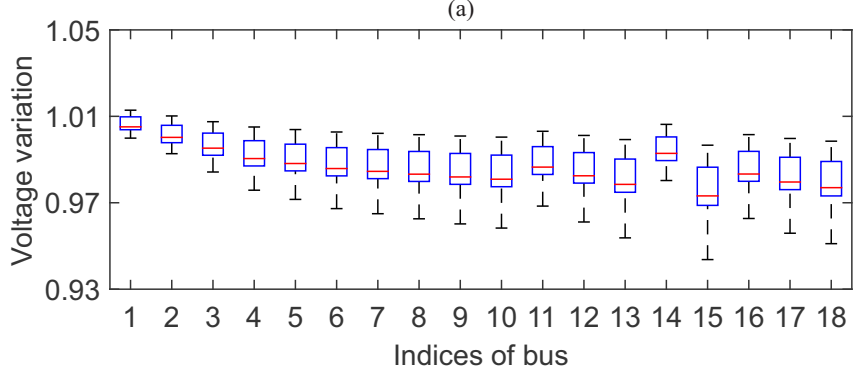

(b)

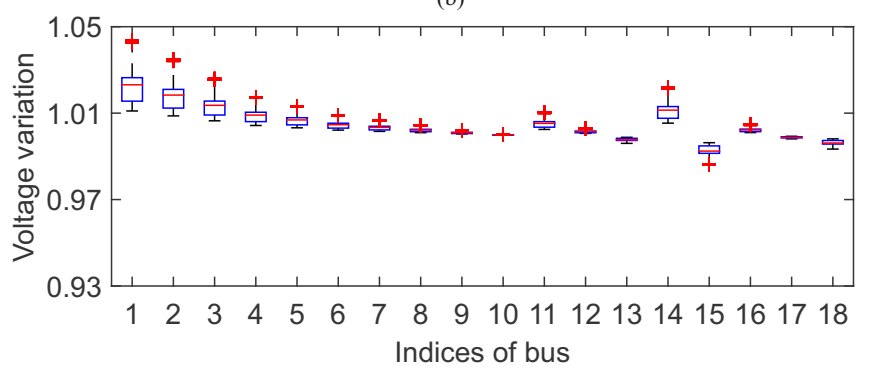

(c)

Fig. 7. LV grid boxplot of the voltage variation along the buses (Phase A) (a). Scenario 1 with CT, (b). Scenario 2 with CT, (c). Both scenarios with ST.

BESS converter size and CT size. In general, smaller values denote better voltage control performance. From Fig. 8 and Fig. 9, the following conclusions are derived:
1) for the same size of the $\mathrm{CT}$, the total voltage variations of $\mathrm{MV}$ and $\mathrm{LV}$ grids reduces with increasing size of converter;

2) with the same converter, when the size of the CT increases, the total voltage variations of the MV and LV grids also increase. This is due to the fact that the larger the size of CT, the more compact the coupling of MV and LV grids, which leads to increased influence from MV grid on LV grid;

3) in the LV network, the voltage performance using the CT with converter is never be as good as it using ST;

4) when the size of the converter is rated to above $1 \mathrm{MVA}$, the voltage control performance of MV grid of new Case 0 is similar to Case 1 . When the converter's rated power is $2 \mathrm{MVA}$, the voltage variations of the LV grid can be reduced within $10 \%$ of its norminal value.
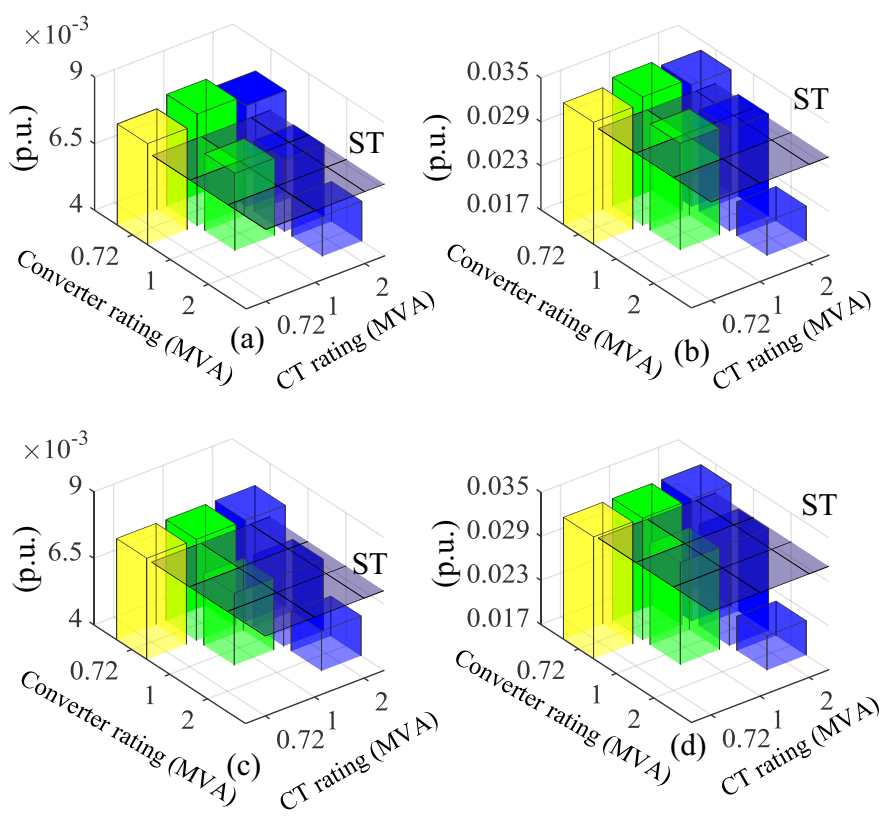

Fig. 8. Voltage performance of the MV grid for new Case 0 (a). Mean voltage variation Scenario 1, (b). Max. voltage variation Scenario 1, (c). Mean voltage variation Scenario 2, (d). Max. voltage variation Scenario 2. The horizontal plane denotes the performance of the ST (Case I).

Tables III and IV summarize the simulation results $(10 \mathrm{sec}-$ onds resolution) of Case 0 and Case 1 for when the components of Case 0 are "1 MVA CT with 1 MVA converter" and "2 MVA CT with 2 MVA converter", respectively. Results in Table III denote that, for the MV network, control performance of Case 0 and Case 1 are similar, whereas - for the LV - Case 0 shows voltage variation above the $10 \%$ threshold. Results in Table IV shows that when increasing the size of the converter to $2 \mathrm{MVA}$, the voltage variations on the LV side are constrained in the allowed range.

\section{Cost comparison}

According to the cost figures in [35], [36], an economic cost estimate of Case 1 (720 kVA ST) results in a $40 \%$ to $60 \%$ increase with respect to Case 0 ( $720 \mathrm{kVA} \mathrm{CT}$ ). Although the larger costs, the simulations results proposed in this paper 

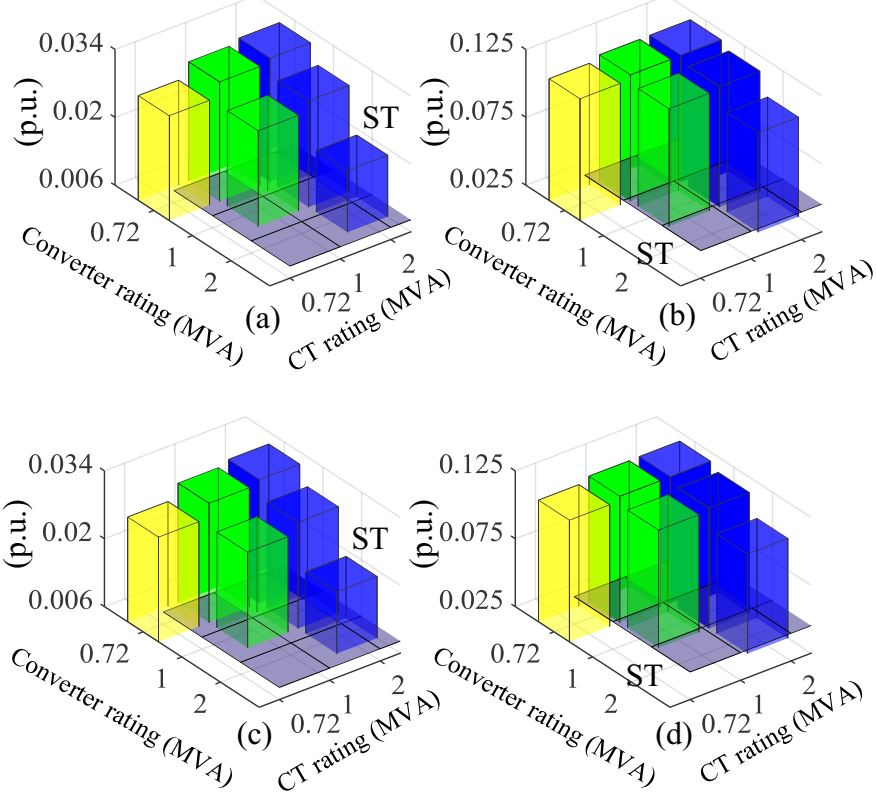

Fig. 9. Voltage performance of the LV grid for new Case 0 (a). Mean voltage variation Scenario 1, (b). Max. voltage variation Scenario 1, (c). Mean voltage variation Scenario 2, (d). Max. voltage variation Scenario 2. The horizontal plane denotes the performance of the ST (Case I).

TABLE III

Voltage Control Performance (CASe 0: 1 MVA CT and 1 MVA CONVERTER)

\begin{tabular}{|c|l|c|c|c|c|}
\hline & Metric & Case 0 MV & Case 1 MV & Case 0 LV & Case 1 LV \\
\hline \multirow{2}{*}{ S1 } & $\bar{m}_{1}$ & $0.69 \%$ & $0.67 \%$ & $2.57 \%$ & $0.85 \%$ \\
& $m_{1}^{\uparrow}$ & $3.15 \%$ & $3.04 \%$ & $11.16 \%$ & $4.53 \%$ \\
\hline \multirow{2}{*}{ S2 } & $\bar{m}_{1}$ & $0.67 \%$ & $0.7 \%$ & $2.49 \%$ & $0.85 \%$ \\
& $m_{1}^{\uparrow}$ & $3.09 \%$ & $3.11 \%$ & $11.03 \%$ & $4.53 \%$ \\
\hline
\end{tabular}

TABLE IV

Voltage Control Performance (CASe 0: 2 MVA CT and 2 MVA CONVERTER)

\begin{tabular}{|l|l|c|c|c|c|}
\hline & Metric & Case 0 MV & Case 1 MV & Case 0 LV & Case 1 LV \\
\hline \multirow{2}{*}{ S1 1} & $\bar{m}_{1}$ & $0.55 \%$ & $0.67 \%$ & $1.87 \%$ & $0.85 \%$ \\
& $m_{1}^{\uparrow}$ & $2.17 \%$ & $3.04 \%$ & $9.84 \%$ & $4.53 \%$ \\
\hline \multirow{2}{*}{ S2 } & $\bar{m}_{1}$ & $0.55 \%$ & $0.7 \%$ & $1.87 \%$ & $0.85 \%$ \\
& $m_{1}^{\uparrow}$ & $2.17 \%$ & $3.11 \%$ & $9.83 \%$ & $4.53 \%$ \\
\hline
\end{tabular}

show that the technical potential of ST with integrated storage for achieving dispatched operation and voltage control to both grid layers is relevant. In the view of the increasing focus on distributed control strategies for power system operations under increased penetration levels of renewable, it is to envisage that suitable deployment policies and economic incentives should be developed to facilitate the adoption of this kind of technology.

\section{CONCLUSIONS}

A control strategy for an MV/LV smart transformer with integrated storage was proposed. On the MV side, it controls i) the active power flow of the power converter to achieve dispatched-by-design operation of the LV feeder according to a profile established the day before operation, and ii) the reactive power injection in order to provide voltage regulation to the MV network on a best effort basis. On the LV side, it controls the voltage at the root of the feeder to assure suitable voltage levels along the line. This configuration was compared against a setup where the MV/LV interface is a conventional transformer and the storage capacity is installed on the LV through a controllable DC/AC power converter. In this case, the converter was controlled to provide dispatched-by-design operation of the active power flow of the LV network and voltage regulation to both the LV and MV network (in a lumped way). The control performance is validated in simulations by considering the MV IEEE 34 nodes test feeder and the CIGRE LV reference network.

A performance assessment of the voltage control performance shows that the proposed control algorithm is able to exploit the capability of the considered setup to decouple the reactive and active power flows. With respect to the base case, it achieves dispatched-by-design operation of the active power flow with a mean absolute error of $0.16 \mathrm{~kW}$ while improving voltage levels by up to $9.3 \%$ and $60.5 \%$ on the MV and LV network with respect to basic case on the MV and LV network, respectively.

In a second simulation analysis, the size of the components of the base case setup was chosen in order to match the voltage control performance as the configuration with the ST. It was shown that to achieve the same performance, a conventional setup would require to oversize the BESS converter by $39 \%$, thus indicating that deploying storage connected to a ST setup might represent an efficient solution to tackle the problem of concurrent provision of ancillary services to the grid.

\section{APPENDIX A}

\section{EXTENSION TO UNBALANCE 3-PHASE NETWORKS}

Let us consider radial power grids whose generic components connected between two of its buses are characterized by circulant shunt admittance and longitudinal impedance matrices (i.e., for a matrix of rank $\mathrm{n}$, its eigenvectors are composed by the roots of unity of order n). For these grids, it is possible to decompose all the nodal/flow voltages, currents and powers with the well-known sequence (or symmetrical components) transformation. The result of the sequence transformation is composed by three symmetrical and balanced three-phase circuits for which the proposed control method can be applied as is. The main problem of this approach, however, is given by the transformation of the voltage/current constraints from the phase domain to the corresponding ones in the sequence domain. Indeed, such a transformation couples the voltage/currents constraints in the sequence domain. However, it is possible to separately bind the zero and negative sequence terms of nodal voltage magnitude and lines current flows by using more conservative constraints as the magnitude of these quantities are restricted by standards/norms (i.e., their maximum magnitudes are known a-priori). The binding of the zero and negative sequences associated with the voltages and currents should allow to decouple the positive sequence. Then, we can apply the proposed control process to the three 
sequences for which we may derive different voltage/current inequalities. Once the three problems are solved, we can transform the obtained voltage/currents/powers in the sequence domain back to the (unbalanced) phase domain.

\section{REFERENCES}

[1] N. Jenkins, R. Allan, P. Crossley, K. D., and S. G., Embedded Generation. The Institution of Electrical Engineers, London, 2000.

[2] J. P. Lopes, N. Hatziargyriou, J. Mutale, P. Djapic, and N. Jenkins, "Integrating distributed generation into electric power systems: A review of drivers, challenges and opportunities," Electric Power Systems Research, vol. 77, no. 9, pp. 1189 - 1203, 2007, distributed Generation.

[3] R. Hidalgo, C. Abbey, and G. Joos, "A review of active distribution networks enabling technologies," in IEEE PES General Meeting, July 2010, pp. 1-9.

[4] K. Christakou, D.-C. Tomozei, M. Bahramipanah, J.-Y. Le Boudec, and M. Paolone, "Primary voltage control in active distribution networks via broadcast signals: The case of distributed storage," IEEE Transactions on Smart Grid, vol. 5, no. 5, pp. 2314-2325, 2014.

[5] F. Sossan, "Equivalent electricity storage capacity of domestic thermostatically controlled loads," Energy, vol. 122, pp. 767 - 778, 2017.

[6] R. Palma-Behnke, C. Benavides, F. Lanas, B. Severino, L. Reyes, J. Llanos, and D. Sáez, "A microgrid energy management system based on the rolling horizon strategy," IEEE Transactions on Smart Grid, vol. 4, no. 2, pp. 996-1006, June 2013.

[7] N. Korada and M. K. Mishra, "Grid adaptive power management strategy for an integrated microgrid with hybrid energy storage," IEEE Transactions on Industrial Electronics, vol. 64, no. 4, pp. 2884-2892, April 2017.

[8] B. Nykvist and M. Nilsson, "Rapidly falling costs of battery packs for electric vehicles," Nature Climate Change, vol. 5, no. 4, pp. 329-332, 2015.

[9] F. Xue, R. Yu, and A. Q. Huang, "A 98.3dc-dc converter for dc microgrid energy storage system applications," IEEE Transactions on Industrial Electronics, vol. PP, no. 99, pp. 1-1, 2017.

[10] L. Wang, D. H. Liang, A. F. Crossland, P. C. Taylor, D. Jones, and N. S. Wade, "Coordination of multiple energy storage units in a low-voltage distribution network," IEEE Transactions on Smart Grid, vol. 6, no. 6, pp. 2906-2918, Nov 2015.

[11] M. Bahramipanah, D. Torregrossa, R. Cherkaoui, and M. Paolone, "A decentralized adaptive model-based real-time control for active distribution networks using battery energy storage systems," IEEE Transactions on Smart Grid, vol. PP, no. 99, pp. 1-1, 2016.

[12] S. J. Lee, J. H. Kim, C. H. Kim, S. K. Kim, E. S. Kim, D. U. Kim, K. K. Mehmood, and S. U. Khan, "Coordinated control algorithm for distributed battery energy storage systems for mitigating voltage and frequency deviations," IEEE Transactions on Smart Grid, vol. 7, no. 3, pp. 1713-1722, May 2016.

[13] Y. S. Kim, E. S. Kim, and S. I. Moon, "Frequency and voltage control strategy of standalone microgrids with high penetration of intermittent renewable generation systems," IEEE Transactions on Power Systems, vol. 31, no. 1, pp. 718-728, Jan 2016.

[14] C. A. Hill, M. C. Such, D. Chen, J. Gonzalez, and W. M. Grady, "Battery energy storage for enabling integration of distributed solar power generation," IEEE Transactions on Smart Grid, vol. 3, no. 2, pp. 850-857, June 2012.

[15] E. Namor, D. Torregrossa, F. Sossan, R. Cherkaoui, and M. Paolone, "Assessment of battery ageing and implementation of an ageing aware control strategy for a load leveling application of a lithium titanate battery energy storage system," in 2016 IEEE 17th Workshop on Control and Modeling for Power Electronics (COMPEL), June 2016, pp. 1-6.

[16] M. A. Abdullah, K. M. Muttaqi, D. Sutanto, and A. P. Agalgaonkar, "An effective power dispatch control strategy to improve generation schedulability and supply reliability of a wind farm using a battery energy storage system," IEEE Transactions on Sustainable Energy, vol. 6, no. 3, pp. 1093-1102, July 2015.

[17] M. Marinelli, F. Sossan, G. T. Costanzo, and H. W. Bindner, "Testing of a predictive control strategy for balancing renewable sources in a microgrid," IEEE Transactions on Sustainable Energy, 2014.

[18] F. Sossan, E. Namor, R. Cherkaoui, and M. Paolone, "Achieving the dispatchability of distribution feeders through prosumers data driven forecasting and model predictive control of electrochemical storage," IEEE Transactions on Sustainable Energy, vol. 7, no. 4, pp. 1762-1777, Oct 2016
[19] H. H. Abdeltawab and Y. A.-R. I. Mohamed, "Mobile energy storage scheduling and operation in active distribution systems," IEEE Transactions on Industrial Electronics, vol. PP, no. 99, pp. 1-1, 2017.

[20] H. Rahimi-Eichi, U. Ojha, F. Baronti, and M. Y. Chow, "Battery management system: An overview of its application in the smart grid and electric vehicles," IEEE Industrial Electronics Magazine, vol. 7, no. 2, pp. 4-16, June 2013.

[21] Q. Wei, D. Liu, F. L. Lewis, Y. Liu, and J. Zhang, "Mixed iterative adaptive dynamic programming for optimal battery energy control in smart residential microgrids," IEEE Transactions on Industrial Electronics, vol. 64, no. 5, pp. 4110-4120, May 2017.

[22] E. Stai, L. Reyes-Chamorro, F. Sossan, J. Y. L. Boudec, and M. Paolone, "Dispatching stochastic heterogeneous resources accounting for grid and battery losses," IEEE Transactions on Smart Grid, vol. PP, no. 99, pp. $1-1,2017$.

[23] M. Liserre, G. Buticchi, M. Andresen, G. De Carne, L. Costa, and Z.$\mathrm{X}$. Zou, "The smart transformer, its impact on the electric grid and its technology challenges," IEEE Trans. Ind. Electr. Mag., in press 2016.

[24] X. She, A. Huang, and R. Burgos, "Review of solid-state transformer technologies and their application in power distribution systems," IEEE Journal of Emerging and Selected Topics in Power Electronics, vol. 1, no. 3, pp. 186-198, Sept 2013.

[25] G. Buticchi, G. D. Carne1, D. Barater, Z. Zou, and M. Liserre, "Analysis of the frequency-based control of a master/slave micro-grid," IET Renewable Power Generation, in press.

[26] X. Yu, X. She, X. Zhou, and A. Q. Huang, "Power management for dc microgrid enabled by solid-state transformer," IEEE Transactions on Smart Grid, vol. 5, no. 2, pp. 954-965, March 2014.

[27] S. Hambridge, A. Q. Huang, and R. Yu, "Solid state transformer (sst) as an energy router: Economic dispatch based energy routing strategy," in 2015 IEEE Energy Conversion Congress and Exposition (ECCE), Sept 2015, pp. 2355-2360.

[28] S. Sarri, M. Paolone, R. Cherkaoui, A. Borghetti, F. Napolitano, and C. A. Nucci, "State estimation of active distribution networks: Comparison between wls and iterated kalman-filter algorithm integrating pmus," in 2012 3rd IEEE PES Innovative Smart Grid Technologies Europe (ISGT Europe). IEEE, 2012, pp. 1-8.

[29] P. A. Pegoraro, J. Tang, J. Liu, F. Ponci, A. Monti, and C. Muscas, "Pmu and smart metering deployment for state estimation in active distribution grids," in Energy Conference and Exhibition (ENERGYCON), 2012 IEEE International. IEEE, 2012, pp. 873-878.

[30] M. Andresen, L. F. Costa, G. Buticchi, and M. Liserre, "Smart transformer reliability and efficiency through modularity," in 2016 IEEE 8th International Power Electronics and Motion Control Conference (IPEMC-ECCE Asia), May 2016, pp. 3241-3248.

[31] K. Christakou, J. Y. LeBoudec, M. Paolone, and D. C. Tomozei, "Efficient computation of sensitivity coefficients of node voltages and line currents in unbalanced radial electrical distribution networks," IEEE Transactions on Smart Grid, vol. 4, no. 2, pp. 741-750, June 2013.

[32] G. D. Carne, M. Liserre, and C. Vournas, "On-line load sensitivity identification in lv distribution grids," IEEE Transactions on Power Systems, vol. PP, no. 99, pp. 1-1, 2016.

[33] W. H. Kersting, "Radial distribution test feeders," in Power Engineering Society Winter Meeting, 2001. IEEE, vol. 2. IEEE, 2001, pp. 908-912.

[34] S. Papathanassiou, N. Hatziargyriou, K. Strunz et al., "A benchmark low voltage microgrid network," in Proceedings of the CIGRE symposium: power systems with dispersed generation, 2005, pp. 1-8.

[35] J. E. Huber and J. W. Kolar, "Volume/weight/cost comparison of a 1mva $10 \mathrm{kv} / 400 \mathrm{v}$ solid-state against a conventional low-frequency distribution transformer," in 2014 IEEE Energy Conversion Congress and Exposition (ECCE), Sept 2014, pp. 4545-4552.

[36] L. F. Costa, G. D. Carne, G. Buticchi, and M. Liserre, "The smart transformer: A solid-state transformer tailored to provide ancillary services to the distribution grid," IEEE Power Electronics Magazine, vol. 4, no. 2, pp. 56-67, June 2017. 


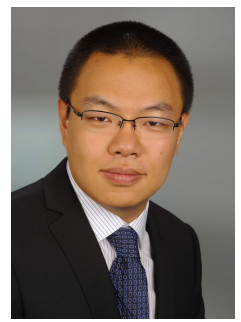

Gao, Xiang ( $\left.S^{\prime} 16\right)$ was born in China. He received He received B.E. in electrical engineering from Southeast University in Nanjing, China, and MSc. in electric power engineering from Royal Institute of Technology (KTH) in Stockholm, Sweden in 2009 and 2012 respectively.

$\mathrm{He}$ has been involved in different projects of analysis of power system stability. $\mathrm{He}$ is a scientific staff member of Chair of Power Electronics, Christian-Albrechts-Universität zu Kiel, Germany since 2016. His main research interest currently is application of power electronics device, e.g. smart transformer, in distribution grid.

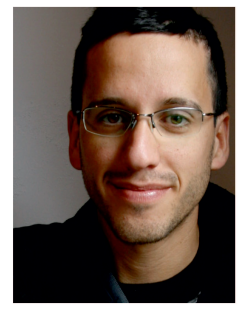

Fabrizio Sossan (M'11) is an Italian citizen and was born in Genova in 1985. He got his M.Sc. in Computer Engineering from the University of Genova in 2010, and, in 2014, the Ph.D. in Electrical Engineering from the Danish Technical University with the thesis Indirect control of flexible demand for power system applications. Since 2015, he is a postdoctoral fellow at EPFL, Switzerland.

His main research interests are modeling and optimization applied to power system.

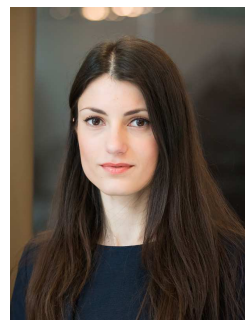

Konstantina Christakou (M'12) was born in Greece, in 1987. In 2010, she completed her undergraduate studies in telecommunications and networking at the National Technical University of Athens, Greece. In 2015 she obtained her Ph.D. degree at École Polytechnique Fédérale de Lausanne (EPFL), Switzerland, where she worked under the joint supervision of Prof. Jean-Yves Le Boudec (LCA2) and Mario Paolone (DESL).

Her research interests include control and real-time operation of electrical grids with special reference to power distribution networks.

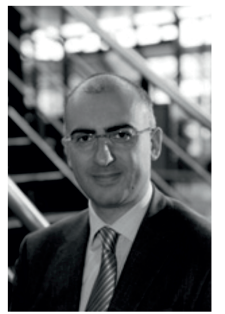

Mario Paolone (M'07-SM'10) received the M.Sc. (Hons.) and Ph.D. degrees in electrical engineering from the University of Bologna, Bologna, Italy, in 1998 and 2002, respectively. In 2005, he was nominated Assistant Professor in power systems at the University of Bologna, where he was with the power systems laboratory until 2011.

$\mathrm{He}$ is currently an Associate Professor at the Swiss Federal Institute of Technology, Lausanne, Switzerland, chair of the Distributed Electrical Systems Laboratory. He was the Co-Chairperson of the Technical Committee of the 9th edition of the International Conference of Power Systems Transients (2009) and Vice-Chair and Chair of the 19th and 20th Power Systems Computation Conference in 2016 and 2018 respectively. He is author or coauthor of more than 220 scientific papers published in reviewed journals and international conferences. He is the Editor-Chief of the Elsevier journal Sustainable Energy, Grids and Networks and the Head of the Swiss Competence Center for Energy Research FURIES. His research interests include power systems with particular reference to realtime monitoring and operation of active distribution networks, integration of distributed energy storage systems, power system protections and power system transients.

In 2013, Prof. Paolone received the IEEE EMC Society Technical Achievement Award.

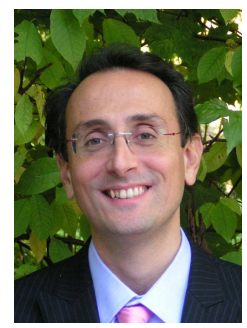

Marco Liserre (S'00-M'02-SM'07-F'13) received the $\mathrm{MSc}$ and $\mathrm{PhD}$ degree in Electrical Engineering from the Bari Polytechnic, respectively in 1998 and 2002. He has been Associate Professor at Bari Polytechnic and Professor at Aalborg University (Denmark).

$\mathrm{He}$ is currently Full Professor and he holds the Chair of Power Electronics at ChristianAlbrechts-Universität zu Kiel (Germany). He has published over 300 technical papers (more than 86 of them in international peer-reviewed journals) and a book. These works have received more than 20000 citations.

Prof. Liserre is listed in ISI Thomson report "The world's most influential scientific minds". He is member of IAS, PELS, PES and IES. He has been serving all these societies in different capacities and he has received 4 IEEE awards and 2 paper awards. 